\title{
Redução Preliminar de Itens da PTS Versão 7 a 14 Anos
}

\author{
Patrícia do Carmo Pereira Ito ${ }^{12}$ \\ Raquel Souza Lobo Guzzo \\ Pontifícia Universidade Católica de Campinas, São Paulo
}

\section{Resumo}

A Pavlovian Temperament Survey - PTS foi desenvolvida para avaliar a expressão comportamental das propriedades do Sistema Nervoso Central. Este estudo teve como objetivo iniciar o processo de aferição e redução de itens da PTS, versão 7 a 14 anos. Participaram da amostra 124 sujeitos de 7 a 14 anos, de ambos os sexos, os quais tiveram suas características temperamentais avaliadas pela PTS. O processo de redução e aferição constou de duas etapas: análise da correlação item total e análise fatorial exploratória. Resultados obtidos indicaram a permanência de 107 itens do total de 252 que inicialmente compunham a escala: 35 itens em FE (38,8\%), 42 em FI (50\%) e 30 em MO (38,4\%). Novos estudos se fazem necessários para uma melhor adequação dos itens ao contexto brasileiro, com ampliação da amostra para dar continuidade ao processo de aferição e redução de itens da PTS versão 7 a 14 anos.

Palavras-chave: Pavlovian Temperament Survey; temperamento; avaliação psicológica.
\end{abstract}

Preliminary Reduction of Items of PTS Version 7 to 14 Years

\begin{abstract}
The Pavlovian Temperament Survey - PTS was developed to evaluate the behavioral expression of Central Nervous System properties. This study aimed to begin the assessment process and reduction of PTS's items, version 7 to 14 years. A sample of 124 subject aged 7 to 14, of both sexes, who had their temperamental characteristics evaluated for PTS took part in the study. The reduction process and assessment consisted of two stages: analysis of the correlation total item and exploratory factorial analysis. The results pointed to the permanence of 107 items of the total of 252 that initially composed the scale: 35 items in FE (38,8\%), 42 in FI (50\%) and 30 in MO (38,4\%). New studies are necessary for a better adaptation of the items to the Brazilian context, with extension of the sample to give continuity to the assessment process and reduction of PTS's items version 7 to 14 years.

Keywords: Pavlovian Temperament Survey; temperament; psychology assessment.
\end{abstract}

O desenvolvimento humano, segundo Teglasi (1998), é o resultado de múltiplos fatores que incluem aspectos: a) psicológicos - pensamentos sobre a avaliação dos eventos (implicações, importância), sentimentos e repertório de comportamentos de enfrentamento; b) biológicos respostas fisiológicas que influenciam processos psicológicos por que elas se traduzem em estados subjetivos de ansiedade, depressão, irritabilidade, inquietação, pensamentos recorrentes ou dificuldades de concentração; e c) grau de apoio social fontes/ recursos para obtenção de apoio para manter ou restabelecer o equilíbrio dos pensamentos, sentimentos e comportamentos funcionais.

O temperamento tem sido caracterizado em diversas teorias como a expressão prematura de diferenças individuais na personalidade, o substrato biológico sob o qual a personalidade se estrutura (Goldsmith \& Rieser-

\footnotetext{
${ }^{1}$ Este estudo foi apresentado na sessão de Comunicações Científicas "Técnicas de Exame Psicológico" da XXXI Reunião Anual de Psicologia, Rio de Janeiro, 2001. Apoio financeiro FAPESP.

${ }^{2}$ Endereço para correspondência: Rua dos Guatás, 250/12B, 13081060 , Campinas, SP. Fone (19) 3208-4519. E-mail: paty_ito@directnet.com.br
}

Danner, 1986; Revelle, 1995; Strelau, 1998, Strelau \& Angleitner, 1991). Considerado um atributo importante para o ajustamento sócio - emocional do indivíduo, teóricos do temperamento afirmam que as crianças tem um estilo comportamental particular que contribui para seu próprio desenvolvimento e sua interação com o ambiente social (McClowry, 1998). Segundo Thomas e Chess (citados em Goldsmith \& cols., 1987), o temperamento deve ser considerado como um fator de influência bidirecional, manifestando-se como "uma resposta a um estímulo externo, oportunidade, expectativa ou demanda" (p. 509). O indivíduo dentro desta visão, é encarado como influenciador ativo de seu ambiente, bem como sujeito a ser influenciado por este (Rutter, 1987). Isto quer dizer que as características temperamentais podem influenciar o tipo de interação que será estabelecida entre o indivíduo e seu ambiente, e a reação das pessoas às características do indivíduo pode por sua vez, gerar um efeito no desenvolvimento da própria pessoa que as provocou (Strelau, 1991). Através do estabelecimento desta "função circular, as características da própria criança promovem uma 
estimulação significativa para seu próprio desenvolvimento", afirma Lerner (1983, p. 240).

Desde longa data, em países da Europa e América do Norte, muitos são os teóricos que se dedicam ao estudo do temperamento e instrumentos para sua avaliação, porém não existe entre estes, um consenso geral sobre sua definição e dimensões, as quais variam em função da abordagem teórica utilizada (Ito \& Guzzo, 2002).

No Brasil, poucos são os estudos desenvolvidos relacionados ao temperamento e instrumentos para sua avaliação. Entre os trabalhos publicados é possível citar os desenvolvidos com a Pavlovian Temperament Survey PTS - versão 14 a 18 anos (Catini, 1999; Guzzo, Primi, Pereira \& Valli, 2000; Guzzo, Riello \& Primi, 1996; Riello, 1999), Pavlovian Temperament Survey - PTS - versão 7 a 14 anos (Catini, 1999; Ito \& Guzzo, 2002), Student Temperament Assessment Record - STAR (Riello, 1992) e Toddler Temperament Scale (Bosa \& Piccinini, 1994). Esta carência de estudos e os trabalhos já iniciados com a escala Pavlovian Temperament Survey - PTS no Brasil motivou este trabalho que apresenta o estudo de aferição e redução de itens da PTS versão 7 a 14 anos.

A PTS, foi desenvolvida com o objetivo de avaliar a expressão comportamental das propriedades do sistema nervoso central, conforme entendido por Pavlov, de modo a permitir estudos transculturais. Tais propriedades foram caracterizadas por Pavlov, do ponto de vista funcional e não fisiológico, reforçando o papel desempenhado por elas no processo de adaptação do indivíduo ao ambiente. As propriedades avaliadas pela escala referem-se a força de excitação (FE), força de inibição (FI) e mobilidade (MO), as quais podem ser consideradas separadamente, cada uma dando origem a uma sub-escala com o mesmo nome da propriedade do sistema nervoso central (Strelau, Angleitner \& Newberry, 1999) .

A PTS, originou-se do Strelau Temperament Inventory STI construído por Strelau em 1969 e utilizado até o início da década de 1990. Neste período, as baixas qualidades psicométricas apresentadas pelo STI, e a convicção de que as características de temperamento investigadas por Pavlov por meio de sua tipologia de sistema nervoso, são constructos cientificamente atrativos e passíveis de estudo, conduziram Strelau, Angleitner, Batelman e Ruch a reverem e reformularem os itens, renomeando o instrumento de Pavlovian Temperament Survey, PTS (Strelau, 1998; Strelau \& Angleitner, 1994; Strelau \& cols., 1999).

$\mathrm{Na}$ construção da escala PTS foi considerado que as propriedades do Sistema Nervoso Central de Pavlov referem-se a traços gerais, revelados em todos os tipos de comportamento, incluindo características motoras, atividades verbais e reações emocionais. A elaboração dos itens que compõem a escala teve início a partir da operacionalização teórica dos constructos força de excitação (FE), força de inibição (FI) e mobilidade (MO) do processo nervoso, constructos considerados etic ${ }^{3}$,isto é, universais entre culturas. Esta operacionalização envolveu a geração de seus componentes de definição (facetas), também comparáveis entre culturas, os quais totalizavam 17 facetas, sete na subescala FE, e cinco para cada uma das outras duas sub-escalas FI e MO. A definição das 17 facetas constituiu a base da geração dos 252 itens que compõem a PTS. Esses itens são considerados universais, comuns para todas as linguagens, e se referem a possíveis situações ou comportamentos nos quais os traços de temperamento podem ser expressos. $\mathrm{O}$ enfoque emic na construção da escala, consistiu em selecionar por meio de elaborados procedimentos psicométricos, os itens mais representativos para cada cultura. Assim, para cada linguagem (cultura), o número e o tipo de itens pode variar (Strelau, 1998; Strelau \& Angleitner, 1994).

Esta estratégia foi aplicada inicialmente para a construção da escala nas versões (países) alemã (Alemanha) e polonesa (Polônia), as quais serviram de base para a versão inglesa (Grã Bretanha e Estados Unidos) estendendo-se posteriormente para as versões da: Austrália, Bélgica, Brasil, Bulgária, Holanda, Grécia, Hungria, Itália, Japão, Coréia, Romênia, Rússia e Espanha. No total, mais de 30 pesquisadores de 16 países estiveram envolvidos nos estudos transculturais da PTS (Strelau \& cols., 1999).

A PTS foi construída inicialmente para avaliar o temperamento de indivíduos acima de 14 anos, Hoogendorp (1992) baseando-se em seus itens, desenvolveu uma nova versão da escala destinada a avaliar o temperamento de crianças e adolescentes de 7 a 14 anos a partir da avaliação de suas mães ou outra pessoa que mantenha contato direto e constante com eles. Nesta nova versão, os itens foram reformulados para que se tornassem representativos de comportamentos de crianças e adolescentes, sendo mantida a mesma fundamentação teórica, número de itens e forma de pontuação da versão destinada a avaliar pessoas maiores de 14 anos.

${ }^{3}$ Berry (citado em Strelau, 1998) menciona dois enfoques básicos relacionados aos estudos transculturais em psicologia, os quais estão presentes também nas avaliações do temperamento entre culturas, nações ou grupos étnicos: 1) enfoque etic - consiste em investigar similaridades ou identidades de um dado fenômeno psicológico ou comportamentos entre diferentes culturas; 2) enfoque emic - destinado a descrever a especificidade do fenômeno psicológico, comportamento ou ambos, dependendo da condição cultural em que os indivíduos são comparados ou grupos que vivem. 
A adaptação da PTS para uso no Brasil iniciou-se em 1994 por meio de projeto enviado pelo Laboratório de Avaliação e Medidas Psicológicas - LAMP, da PUC Campinas, ao $\mathrm{CNPq}$ intitulado "Temperamento: Construção de uma escala para avaliação do Temperamento no Brasil" (Guzzo, 1997) destinado a adaptar a versão da escala para indivíduos maiores de 14 anos. Em 1996, teve início no LAMP a adaptação da PTS versão 7 a 14 anos, cujo projeto enviado ao CNPq chamava-se "Construção da versão infantil da escala PTS para a realidade brasileira: estudo preliminar" (Guzzo, 1997).

A versão para indivíduos maiores de 14 anos teve suas qualidades psicométricas avaliadas e foi reduzida de 252 itens para 57 itens, permanecendo os itens mais representativos para a realidade brasileira (Guzzo \& cols., 1996). Aplicações posteriores com a escala em sua versão reduzida, coletaram dados para a normatização do instrumento (Guzzo \& cols., 2000).

A PTS versão 7 a 14 anos, em seus estudos iniciais (Catini, 1999; Ito e Guzzo, 2002), demonstrou que o instrumento possui boas qualidades psicométricas, porém uma queixa constante dos participantes destes estudos é a de que o instrumento é demasiado longo, com itens repetitivos. Considerando esta queixa dos pais, o fato de que a escala foi construída com o intuito de realizar estudos transculturais sobre o temperamento, e já prevendo uma redução para permanência dos itens mais representativos para cada cultura, o presente estudo objetiva iniciar o processo de aferição dos itens da escala, por meio da seleção dos itens mais representativos para a realidade brasileira.

\section{Método}

\section{Participantes}

A amostra foi composta por 124 participantes de ambos os sexos, com idades variando de 7 a 14 anos,

Tabela 1

Distribuição dos Sujeitos Considerando o Sexo e a Idade

\begin{tabular}{llll}
\hline & Feminino & Masculino & Total \\
\hline 7 anos & 12 & 10 & 22 \\
8 anos & 6 & 10 & 16 \\
9 anos & 13 & 7 & 20 \\
10 anos & 6 & 10 & 16 \\
11 anos & 6 & 4 & 10 \\
12 anos & 7 & 5 & 12 \\
13 anos & 8 & 13 & 21 \\
14 anos & 0 & 7 & 7 \\
\hline Total & 58 & 66 & 124 \\
\hline
\end{tabular}

Psicologia: Reflexão e Crítica, 2003, 16(1), pp. 183-190 estudantes da $1^{a}$ a $8^{a}$ série do Ensino Fundamental, que tiveram suas características de temperamento avaliadas por suas mães ou outro responsável que conhecesse bem a criança ou adolescente (Tabela 1). Os participantes eram provenientes da cidade de Campinas e região.

\section{Material}

A PTS versão 7 a 14 anos é composta por 252 itens, que avaliam três fatores, baseados nas propriedades pavlovianas do Sistema Nervoso Central: Força de Excitação (FE), Força de Inibição (FI) e Mobilidade (MO), os quais constituem três sub-escalas designadas pelo mesmo nome do fator. Cada um dos fatores é composto por diferentes facetas: FE - sete facetas; FI e MO cinco facetas cada um, que apresentam diferentes números de itens (Tabela 2) (Strelau \& cols., 1999). A descrição de fatores e suas respectivas facetas é feita a seguir.

O fator força de excitação (FE) está presente em 90 itens da escala, e representa a capacidade do indivíduo manter-se sem inibição diante de uma situação de estimulação intensa e prolongada. Suas facetas são: FE1 - situações ameaçadoras não restringem uma atividade ou ação anteriormente planejada; FE2 - propensão a empreender atividades/ ações em situações altamente estimulantes; FE3 - preferência em realizar atividades exigentes e/ ou arriscadas; FE4- ausência de alterações ou distúrbios emocionais no desempenho de atividades sob pressão física e/ ou social; FE5 - manutenção da eficiência de desempenho diante de atividades e situações de alto valor estimulativo; FE6 - resistência à fadiga quando da realização de atividades longas e/ou intensivas; FE7 - capacidade de reagir adequadamente sob forte tensão emocional.

O fator força de inibição (FI) está incluído em 84 itens, e refere-se a capacidade do indivíduo interromper um determinado comportamento quando necessário, ou efetuar uma mudança de reação. Sua facetas são: FI1 - capacidade de refrear comportamentos que por razões sociais não são esperados; FI2 - não dificuldade em esperar pela execução de uma tarefa quando um atraso é previsto; FI3 capacidade de interromper a realização de uma tarefa ou reação a uma dada situação, quando necessário; FI4 capacidade de adiar reações se isto é requerido pelas circunstâncias; FI5 - capacidade de refrear a expressão da emoção quando a situação requer.

A mobilidade (MO) é definida como a habilidade de responder adequadamente a contínuas mudanças no ambiente, e está presente em 78 itens. Sua facetas são:MO1 - reação adequada a mudanças inesperadas no meio; MO2 - adaptação rápida a novos ambientes; MO3 - passagem rápida de uma atividade para outra; MO4 - agilidade na mudança de humor positivo - negativo e vice versa, 
Tabela 2

Distribuição dos Itens da PTS Considerando Fatores e Facetas

\begin{tabular}{|c|c|c|c|}
\hline Fator & Faceta & Itens & $\mathrm{N}^{\circ}$ itens \\
\hline & FE1 & $1,4,7,10,13,16,19,22,25,28,31,221$ & 12 \\
\hline & FE2 & $34,37,40,43,46,49,52,55,58,61,64,67,233$ & 13 \\
\hline FE & FE3 & $70,73,76,79,82,85,88,91,94,97,100,103,106,109,112,115$ & 16 \\
\hline \multirow[t]{5}{*}{$N=90$} & FE4 & $118,121,124,127,130,133,136,139,217,245$ & 10 \\
\hline & FE5 & $142,145,148,151,154,157,160,163,166,169,172,175,178,181$ & 14 \\
\hline & FE6 & $184,187,190,193,196,199,203,207,211,215,219$ & 11 \\
\hline & FE7 & $201,205,209,213,223,226,228,230,236,240,243,247,249,252$ & 14 \\
\hline & FI1 & $2,5,8,11,14,17,20,23,26,29,32,164,167,170,173,176,250$ & 17 \\
\hline FI & FI2 & $35,38,41,44,47,50,53,56,59,62,65,68,179,182,185,234$ & 16 \\
\hline \multirow{4}{*}{$N=84$} & FI3 & $71,74,77,80,83,86,89,92,95,98,101,104,107,110,113,251$ & 16 \\
\hline & FI4 & $\begin{array}{l}116,119,122,125,128,131,134,137,140,143,152,155,158,161 \\
188,191,194,239\end{array}$ & 18 \\
\hline & FI5 & $\begin{array}{l}146,149,197,200,204,208,212,216,220,224,227,231,232,237, \\
241,244,248\end{array}$ & 17 \\
\hline & MO1 & $3,6,9,12,15,18,21,24,27,30,33,162,165,168,171,174,177,238$ & 18 \\
\hline \multirow[t]{4}{*}{$\begin{array}{l}\mathrm{MO} \\
N=78\end{array}$} & MO2 & $\begin{array}{l}36,39,42,45,48,51,54,57,60,63,66,69,180,183,186,189,192, \\
229,242\end{array}$ & 19 \\
\hline & MO3 & $\begin{array}{l}72,75,78,81,84,87,90,93,96,99,102,105,108,111,114,117,235 \text {, } \\
246\end{array}$ & 18 \\
\hline & MO4 & $120,123,126,129,132,135,138,141,144,153,156,159$ & 12 \\
\hline & MO5 & $147,150,195,198,202,206,210,214,218,222,225$ & 11 \\
\hline
\end{tabular}

dependendo da situação; MO5 - preferência por situações que requerem diferentes atividades a serem realizadas simultaneamente.

Os 252 itens da PTS versão 7 a 14 anos são avaliados pelo formato Likert, com quatro possibilidades de resposta: concordo plenamente, concordo, discordo e discordo plenamente, com a pontuação variando de um a quatro pontos. Os itens podem ser positivos ou negativos, sendo que, quando negativos, deve ser procedida a inversão dos pontos do item no momento da avaliação.

\section{Procedimento}

No que se refere a aplicação da PTS, esta foi feita em entrevistas individuais, onde as mães eram solicitadas a responderem aos itens da escala de acordo com as características percebidas de sua criança ou adolescente.

Tabela 3

Consistência Interna dos Fatores Avaliados pela PTS

\begin{tabular}{cl}
\hline Fator & $\alpha$ \\
\hline FE & 0,89 \\
FI & 0,90 \\
MO & 0,88 \\
\hline
\end{tabular}

\section{Resultados}

Num primeiro momento, antes de dar início ao processo de redução dos itens, os dados coletados foram submetidos ao cálculo do coeficiente alpha de Cronbach, o qual evidenciou índices de consistência interna bastante satisfatórios nos três fatores avaliados pelos instrumento (Tabela 3).

Para iniciar o processo de redução dos itens da escala, foi feito o cálculo da correlação item total para cada um dos fatores avaliados pela PTS, objetivando verificar quais os itens mais consistentes. De acordo com critérios constantes no manual da escala PTS (Strelau \& cols., 1999), permaneceriam os itens com correlação item - total igual ou superior a 0,15 . Resultados obtidos nesta análise (Tabela 4) evidenciam que todas as facetas permaneceram representadas em seus respectivos fatores com porcentagens de permanência de itens variando de $58,3 \%$ a $100 \%$, sendo que FE permaneceu em seu total com $78,8 \%$ de seus itens iniciais, FI com $80,9 \%$ e MO com $76,9 \%$ dos seus itens.

A próxima etapa no processo de redução da escala, foi submeter os itens que permaneceram após a análise da correlação item - total à análise fatorial. Foi realizada uma análise fatorial exploratória utilizando o método de 
Tabela 4

Itens que Permaneceram na PTS Considerando-se o Critério de Inclusão Correlação Item Total Igual ou Superior a 0,15

\begin{tabular}{|c|c|c|c|c|}
\hline Fator & Faceta & Itens & $\mathrm{N}^{\circ}$ itens & $\begin{array}{c}\% \text { itens } \\
\text { permaneceram }\end{array}$ \\
\hline \multirow{8}{*}{$\mathrm{FE}$} & FE1 & $1,10,13,16,19,25,28$ & 7 & 58,3 \\
\hline & FE2 & $37,40,43,55,61,64,67,233$ & 8 & 61,5 \\
\hline & FE3 & $73,79,82,88,91,94,97,100,103,106,109,112,115$ & 13 & 81,2 \\
\hline & FE4 & $118,124,127,130,133,136,139,217,245$ & 9 & 90 \\
\hline & FE5 & $142,145,148,151,154,157,160,163,166,169,172,175,178,181$ & 14 & 100 \\
\hline & FE6 & $184,187,193,196,199,211,215,219$ & 8 & 72,7 \\
\hline & FE7 & $201,205,209,213,226,228,230,236,240,247,249,252$ & 12 & 85,7 \\
\hline & Total & & 71 & 78,8 \\
\hline \multirow{6}{*}{ FI } & FI1 & $2,5,8,11,14,17,20,23,26,29,32,164,167,173,176,250$ & 16 & 94,1 \\
\hline & FI2 & $35,41,47,50,53,56,59,62,65,68,182,185,234$ & 13 & 81,2 \\
\hline & FI3 & $71,74,77,86,89,95,98,101,104,107,110$ & 11 & 68,7 \\
\hline & FI4 & $116,119,125,131,134,140,143,152,155,158,161,188,191,239$ & 14 & 77,7 \\
\hline & FI5 & $149,197,200,204,208,212,220,224,227,231,237,241,244,248$ & 14 & 82,3 \\
\hline & Total & & 68 & 80,9 \\
\hline \multirow{6}{*}{ MO } & MO1 & $3,6,9,12,15,18,21,30,33,162,165,171,174,177,238$ & 15 & 83,3 \\
\hline & MO2 & $36,42,45,48,57,60,63,66,69,180,183,189,229,242$ & 14 & 73,6 \\
\hline & MO3 & $75,78,81,87,93,96,99,102,105,108,111,114,117,235,246$ & 15 & 83,3 \\
\hline & MO4 & $120,126,129,132,138,141,144,153,156$ & 9 & 75 \\
\hline & MO5 & $147,195,202,206,210,214,222$ & 7 & 63,9 \\
\hline & Total & & 60 & 76,9 \\
\hline Total & & & 199 & 78,9 \\
\hline
\end{tabular}

Tabela 5

Exemplos de Itens com Cargas Fatoriais Baixas em seus Respectivos Fatores, Eliminados da Escala

\begin{tabular}{|c|c|c|c|c|}
\hline \multirow[t]{2}{*}{ Fator/Faceta } & \multirow[t]{2}{*}{ Item } & \multicolumn{3}{|c|}{ Cargas Fatoriais/Componentes } \\
\hline & & $1(\mathrm{FI})$ & $2(\mathrm{MO})$ & $3(\mathrm{FE})$ \\
\hline FE1 & $\begin{array}{l}\text { 028) É capaz de começar a cantar em uma festa, mesmo } \\
\text { percebendo que ninguém vai prestar atenção }\end{array}$ & 0,021 & 0,140 & 0,098 \\
\hline FE2 & 233) Acha ruim falar em público & 0,030 & 0,325 & $-0,013$ \\
\hline FE6 & 193) Precisa de paradas em longas viagens de carro & 0,066 & 0,238 & 0,027 \\
\hline FI3 & $\begin{array}{l}\text { 110) Pode, facilmente, interromper uma atividade quando } \\
\text { precisa começar outra }\end{array}$ & 0,099 & 0,462 & $-0,042$ \\
\hline FI4 & $\begin{array}{l}\text { 158) Espera uma oportunidade adequada para contar } \\
\text { alguma coisa importante para alguém }\end{array}$ & 0,078 & 0,392 & 0,058 \\
\hline FI5 & $\begin{array}{l}\text { 200) Comporta-se, educadamente, mesmo com pessoas } \\
\text { que não lhe agradam, mas que não pode evitar }\end{array}$ & 0,078 & 0,568 & 0,052 \\
\hline MO1 & 006) Atrapalha-se diante de um imprevisto & 0,203 & 0,065 & 0,224 \\
\hline MO3 & 078) Volta às aulas depois das férias, sem incomodar-se & 0,295 & 0,077 & 0,337 \\
\hline MO3 & $\begin{array}{l}\text { 081) Precisa de alguns minutos para se levantar quando } \\
\text { acorda }\end{array}$ & 0,328 & 0,008 & 0,170 \\
\hline
\end{tabular}


Tabela 6

Exemplos de Itens com Cargas Fatoriais Elevadas em seus Respectivos Fatores, que Permaneceram na Escala

\begin{tabular}{|c|c|c|c|c|}
\hline \multirow{2}{*}{$\begin{array}{l}\text { Fator/ } \\
\text { Faceta }\end{array}$} & \multirow[t]{2}{*}{ Item } & \multicolumn{3}{|c|}{ Cargas Fatoriais/Componentes } \\
\hline & & $1(\mathrm{FI})$ & $2(\mathrm{MO})$ & $3(\mathrm{FE})$ \\
\hline FE5 & 163) Sua capacidade diminui sob pressão & 0,071 & 0,106 & 0,727 \\
\hline FE5 & $\begin{array}{l}\text { 178) Seu rendimento fica prejudicado, quando entra em rivalidade com } \\
\text { outras crianças }\end{array}$ & 0,093 & 0,242 & 0,595 \\
\hline FE7 & 228) Apresenta dificuldades para falar em situações de decisão & 0,108 & 0,144 & 0,668 \\
\hline FI1 & 020) Prefere seguir suas próprias idéias, aos invés de obedecer regras & 0,594 & 0,167 & $-0,072$ \\
\hline FI2 & 059) Irrita-se quando uma atividade que gosta é interrompida & 0,695 & $-0,122$ & 0,132 \\
\hline FI4 & 239) Sente dificuldade de esperar até que você atenda um pedido dele & 0,674 & 0,028 & $-0,151$ \\
\hline MO1 & $\begin{array}{l}\text { 033) Acostuma-se, facilmente, a algum outro lugar quando não pode ir } \\
\text { ao de sempre }\end{array}$ & $-0,035$ & 0,604 & $-0,006$ \\
\hline MO3 & 102) Pode trocar de atividades, facilmente & $-0,037$ & 0,659 & $-0,053$ \\
\hline MO4 & $\begin{array}{l}\text { 120) Pode esquecer sua raiva, sem esforço, se briga com alguém e mais } \\
\text { tarde encontra essa pessoa }\end{array}$ & $-0,112$ & 0,587 & 0,118 \\
\hline
\end{tabular}

Tabela 7

Itens que Permaneceram na PTS após Exclusão pela Análise Fatorial

\begin{tabular}{|c|c|c|c|c|}
\hline Fator & Faceta & Itens & $\mathrm{N}^{\mathrm{o}}$ itens & $\begin{array}{c}\% \text { itens } \\
\text { permaneceram }\end{array}$ \\
\hline \multirow{8}{*}{ FE } & FE1 & $13,16,19$ & 3 & 25 \\
\hline & FE2 & $37,43,61,64,67$ & 5 & 38,4 \\
\hline & FE3 & $73,79,88,94,97,103,109,112,115$ & 9 & 56,2 \\
\hline & FE4 & $118,124,127,130,133$ & 5 & 50 \\
\hline & FE5 & $151,157,160,163,166,169,178,181$ & 8 & 57,1 \\
\hline & FE6 & $184,211,215$ & 3 & 27,2 \\
\hline & FE7 & 226,228 & 2 & 14,2 \\
\hline & Total & & 35 & 38,8 \\
\hline \multirow{6}{*}{ FI } & FI1 & $2,8,11,14,20,23,26,29,32,250$ & 10 & 58,8 \\
\hline & FI2 & $41,50,53,56,59,62,65,68,182$ & 9 & 56,2 \\
\hline & FI3 & $71,77,89,95,98$ & 5 & 31,2 \\
\hline & FI4 & $119,131,140,143,152,155,191,239$ & 8 & 44,4 \\
\hline & FI5 & $149,204,208,212,220,224,227,237,241,248$ & 10 & 58,8 \\
\hline & Total & & 42 & 50 \\
\hline \multirow{6}{*}{$\mathrm{MO}$} & MO1 & $9,12,33,165$ & 4 & 22,2 \\
\hline & MO2 & $36,45,48,60,63,66,69,180,183,189,229,242$ & 12 & 63,1 \\
\hline & MO3 & $75,87,99,102,105,114,246$ & 7 & 38,8 \\
\hline & $\mathrm{MO} 4$ & $120,129,132,138,144$ & 5 & 41,6 \\
\hline & MO5 & 147,202 & 2 & 18,1 \\
\hline & Total & & 30 & 38,4 \\
\hline Total & & & 107 & 42,4 \\
\hline
\end{tabular}


rotação varimax, com um número determinado de três fatores para a extração. Como critério para permanência do item, foi adotado que o mesmo deveria apresentar uma carga fatorial maior que 0,20 no fator onde os itens estivessem aglutinados e uma diferença de 0,10 para com os outros fatores. Considerando este critério, as cargas fatoriais mínimas obtidas pelos itens nos fatores foram $\mathrm{FE}=-0,013$ (item 233); $\mathrm{MO}=0,008$ (item 081); FI = 0,078 (item 158) (Tabela 5) e as cargas fatoriais máximas obtidas foram $\mathrm{FE}=0,727$ (item 163); FI $=0,695$ (item 059); $\mathrm{MO}=0,659$ (item 102) (Tabela 6).

Tabela 8

Resultados Obtidos pelos Fatores Componentes após Eliminação dos Itens pelo Critério de Exclusão da Análise Fatorial

\begin{tabular}{llll}
\hline Componentes & $1(\mathrm{FI})$ & $2(\mathrm{MO})$ & $3(\mathrm{FE})$ \\
\hline Eigenvalue & 10,32 & 8,83 & 8,82 \\
\% da variância & 9,64 & 8,26 & 8,24 \\
alpha & 0,90 & 0,89 & 0,88 \\
\hline
\end{tabular}

O critério de permanência de itens adotado na etapa da análise fatorial conservou 107 itens distribuídos entre as 17 facetas que compõem os três fatores avaliados pela escala PTS (Tabela 7).

Após esta etapa, os itens foram novamente submetidos a análise fatorial, e novamente os itens se agruparam de maneira semelhante, com eigenvalue de 10,32 no fator FI, 8,83 em MO e 8,82 em FE. O fator FI foi responsável por $9,64 \%$ da variância total observada, $\mathrm{MO}$ por 8,26 e FE por 8,24. O índice de consistência interna dos fatores calculado pelo alpha de Cronbach mantevese satisfatório nos três fatores (Tabela 8)

Nesta nova análise fatorial foi possível observar que alguns itens, dos três fatores, apresentaram cargas fatoriais baixas, inferiores a 0,20 ou cargas fatoriais em mais de um fator.

\section{Discussão}

O processo de aferição e redução de itens da PTS versão 7 a 14 anos, conforme previsto no manual da escala (Strelau \& cols., 1999), apresentou-se bem sucedido, uma vez que, mesmo após a eliminação de itens, os fatores que compõem a escala continuaram apresentando índices de consistência interna satisfatórios.

Todas facetas e seus respectivos fatores continuaram presentes na escala, algumas com mais outras com menos itens. A permanência de números diferenciados de itens nas facetas e fatores é um aspecto esperado conforme literatura deste instrumento (Strelau, 1998; Strelau \& Angleitner, 1994;
Strelau \& cols., 1999), uma vez que a PTS foi construída com um pool de 252 itens para que, por meio de elaborados orocedimentos psicométricos, sejam selecionados os itens mais representativos para a cultura na qual a escala está sendo adaptada e estudada.

Comparada a versões reduzidas em outros países, a PTS versão 7 a 14 anos continua extensa, podendo ter mais itens eliminados. A versão que apresenta menor número de itens é da Romênia com um instrumento de 48 itens (16 itens em cada fator - FE, FI e MO), a versão que apresenta maior número de itens é a da Alemanha, são 72 itens, 24 para cada um dos fatores avaliados (Strelau \& cols., 1999). No Brasil, a versão da PTS destinada a avaliar o temperamento de indivíduos maiores de 14 anos após o processo de seleção de itens, resultou num instrumento com 57 itens, 19 avaliando cada um dos fatores (Guzzo \& cols., 1996).

Análise das cargas fatoriais obtidas pelos itens nos fatores após a seleção de itens pelo critério da análise fatorial evidenciaram que alguns itens apresentaram cargas fatoriais baixas em seus fatores de origem, e/ou apresentavam cargas fatoriais em outro fator além daquele ao qual pertencia. Tal resultado conduz a necessidade de rever a formulação dos itens, tornando-os mais representativos de seus fatores e adequados ao contexto sócio cultural brasileiro.

Novas aplicações e uma conseqüente ampliação da amostra se fazem necessárias, para realização de novos estudos das qualidades psicométricas da escala, bem como para dar continuidade ao processo de redução de itens da PTS versão 7 a 14 anos.

\section{Referências}

Bosa, C. A. \& Piccinini, C. A. (1994). Temperamento e apego: A questão da ortogonalidade em foco. Arquivos Brasileiros de Psicologia, 46(3/4), 95118.

Catini, N. (1999). Temperamento: Estudo inicial da escala PTS infantil. Dissertação de Mestrado não-publicada, Curso de Pós-graduação, Pontifícia Universidade Católica de Campinas. Campinas, São Paulo.

Goldsmith, H. H., Buss, A. H., Plomin, R., Rothbart, M. K., Chess, S., Thomas, A., Hinde, R. A. \& McCall, R. B. (1987). Rountdtable: What is temperament? Four approaches. Child Development, 58, 505-529.

Goldsmith, H. H. \& Rieser-Danner, L. A. (1986). Variation among temperament theories and validation studies of temperament assessment. Em G. A. Kohnstamm (Org.), Temperament discussed: Temperament and development in infancy and childhood (pp. 1-10). Lisse: Swets \& Zeitlinger.

Guzzo, R. S. L. (1997). Escala de temperamento e realidade brasileira: Estudo do Pavlovian Temperament Survey (Relatório técnico de pesquisa/ $\mathrm{CNPq}$ setembro/1996 a agosto/1997).

Guzzo, R. S. L., Primi, R., Pereira, P. C. \& Valli, C. M. M. (2000). Características psicométricas da PTS - versão adulto: Validade, precisão e padronização para a realidade brasileira. (Material não-publicado)

Guzzo, R. S. L., Riello, I. C. \& Primi, R. (1996). Pavlovian Temperament Survey - PTS: Análise de itens e teste de realidade. Psicologia Escolar e Educacional, 1(1), 53-59. 
Hoogendorp, N. (1992). Temperament bei kindern: Die entwicklung und überprünfung einer kinderform des PTS (Temperamento de crianças: o desenvolvimento e avaliação de uma versão infantil da PTS). Diplomarbeit im Fakultät für psychologie und Sportwissenschaft na Der Universität Bielefeld.

Ito, P. C. P. \& Guzzo, R. S. L. (2002). Diferenças individuais: Temperamento e personalidade; importância da teoria. Estudos de Psicologia, 19(1), $91-$ 100.

Ito, P. C. P. \& Guzzo, R. S. L. (2002). Temperamento: Características e determinação genética. Psicologia: Reflexão e Crítica, 15(2), 425-436.

Lerner, J. V. (1983). The role of temperament in psychosocial adaptation in early adolescents: A test of a "Goodness-of-fit" model. Journal of Genetic Psychology, 143, 149-157.

McClowry, S. G. (1998). The science and art of using temperament as the basis for intervention. School Psychology Review, 24(4), 551-563.

Revelle, W. (1995). Personality process. Annual Review of Psychology, 46, 295328 .

Riello, I. C. (1992). O temperamento de adolescentes na realidade brasileira: Um estudo preliminar para a avaliação de uma escala. Disssertação de Mestrado nãopublicada, Curso de Pós Graduação, Pontifícia Universidade Católica de Campinas. Campinas, São Paulo.

Riello, I. C. (1999). Temperamento: Perfil de adolescentes com diferentes competências em natacão. Tese de Doutorado não-publicada, Curso de Pós Graduação, Pontifícia Universidade Católica de Campinas. Campinas, São Paulo.

Rutter, M. (1987). Temperament, personality and personality disorder. British Journal of Psychiatry, 150, 443-458.
Strelau, J. (1991, Julho - Agosto). Temperament and giftedness in children and adolescents. Conferência apresentada na "Nineth world conference on gifted and talented children" - Hage, Netherlands.

Strelau, J. (1998). Temperament: A psychological perspective. New York: Plenum. Strelau, J. \& Angleitner, A. (1991). Temperament research: Some divergences and similarities. Em J. Strelau \& A. Angleitner (Orgs.), Explorations in temperament: International perspective on theory and measurement (pp. 1-12). New York: Plenum.

Strelau, J. \& Angleitner, A. (1994). Cross cultural studies on temperament: Theoretical considerations and empirical studies based on the Pavlovian Temperament Survey. Personality and Individual Differences, 16(2), 331-342.

Strelau, J., Angleitner, A. \& Newberry, B. H (1999). The Pavlovian Temperament Survey (PTS): An International Handbook. Seattle: Hogrefe \& Huber.

Teglasi, H. (1998). Introduction to the mini-series: Implications of temperament for the practice of school psychology. School Psychology Review, 24(4), 475-478.

Sobre as autoras

Patrícia do Carmo Pereira Ito é Mestre em Psicologia Escolar e Doutoranda em Psicologia na Pontifícia Universidade Católica de Campinas. Integrante da equipe do Laboratório de Avaliação e Medidas Psicológicas - LAMP. Bolsista FAPESP.

Raquel Souza Lobo Guzzo é Professora Titular do Instituto de Psicologia e Fonoaudiologia da Pontifícia Universidade Católica de Campinas. Coordenadora do Laboratório de Avaliação e Medidas Psicológicas - LAMP. Doutora em Psicologia Escolar pela Universidade de São Paulo. Pesquisadora do CNPq. 